\title{
An Ultra-Flexible Modular High Vacuum Setup for Thin Film Deposition
}

Thomas Götsch, 1, 2,3, a) Eva-Maria Wernig, ${ }^{1, \text { a) }}$ Bernhard Klötzer, ${ }^{1}$ Thomas Schachinger, ${ }^{4}$

Julia Kunze-Liebhäuser, ${ }^{1}$ and Simon Penner ${ }^{1, b)}$

1) Department of Physical Chemistry, University of Innsbruck, Innrain 52c,

A-6020 Innsbruck, Austria

2) Department of Heterogeneous Reactions, Max-Planck-Institute for Chemical

Energy Conversion, Stiftstraße 34-36, 45470 Mülheim an der Ruhr,

Germany

3) Department of Inorganic Chemistry, Fritz-Haber Institute

of the Max-Planck Society, Faradayweg 4-6, 14195 Berlin,

Germany

4) University Service Center for Transmission Electron Microscopy,

TU Wien, Wiedner Hauptstraße 8-10, A-1040 Vienna,

Austria

This article may be downloaded for personal use only. Any other use requires prior permission of the author and AIP Publishing. This article appeared in Review of Scientific Instruments 90 (2019) 023902-1 - 023902-13

and may be found at

DOI: $10.1063 / 1.5065786$ 
A modular high vacuum chamber dedicated to thin film deposition is presented. We detail the vacuum and gas infrastructure required to operate two highly flexible chambers simultaneously, with a focus on evaporation techniques (thermal and electron beam) and magnetron sputtering, including baking equipment to remove residual water from the chamber. The use of O-ring-sealed flat flanges allows a tool-free assembly process, in turn enabling rapid changes of the whole setup. This leads to a high flexibility regarding the deposition techniques as the chamber can be adapted to different sources within minutes, permitting the formation of multilayer systems by consecutive depositions onto the same substrate. The central piece of the chamber is a flat flange ground glass tube or cross. The glass recipient permits optical monitoring of the deposition process. Further equipment, such as for the introduction of gases, additional pressure gauges or evaporators, can be incorporated via specifically designed stainless steel/aluminum interconnector and blank flanges. In the end, we demonstrate the preparation of an unsupported thin film system consisting of electron-beam-evaporated platinum nanoparticles embedded in magnetron-sputtered zirconia $\left(\mathrm{ZrO}_{2}\right)$, deposited onto $\mathrm{NaCl}$ single crystals, which subsequently can be removed by dissolution. These films are further analyzed by means of transmission electron microscopy, X-ray photoelectron spectroscopy and atomic force microscopy.

a) T.G. and E.-M.W. contributed equally.

b) Electronic mail: simon.penner@uibk.ac.at 


\section{INTRODUCTION}

Thin films are becoming ever more important for a multitude of technical applications. For instance, they can be used for optical coatings, ${ }^{1-4}$ as thermal barrier coatings,${ }^{5}$ to increase the hardness of, for example, mechanical tools ${ }^{6}$ as well as for medical applications ${ }^{7}$ and gas sensors. ${ }^{8,9}$

These thin film applications are increasingly finding their way into the energy sector: a significant field of research is devoted to solar cells based on thin films, ${ }^{10-13}$ as well as thin film batteries ${ }^{14}$ or fuel cell catalysts. ${ }^{15,16}$ A relatively new approach to lowering the operating temperatures of solid oxide fuel cells (SOFCs), which remains one of the challenges to their widespread adoption, ${ }^{17}$ heavily relies on thin films: In these so-called micro-SOFCs, the thicknesses of the electrolyte and the electrodes are decreased to the $100 \mathrm{~nm}$ regime by employing various thin film deposition techniques, leading to higher ionic conductivities at lower temperatures. ${ }^{18,19}$ Additionally, thin films have relevance as electrocatalysts and (photo)catalysts for a variety of processes. ${ }^{20-23}$ Furthermore, the current trend of minia-

turization of electronic devices results in, e.g., thin film capacitors, ${ }^{24,25}$ transistors $^{26-29}$ or memory and storage devices. ${ }^{30-32}$

Another important application of thin films are model systems for fundamental research in catalysis. ${ }^{33,34}$ The advantages of these model catalysts lie in the fact that it is possible to embed metal particles into a support (e.g. an oxide) for investigations of the metal-support interaction under realistic conditions by means of electron microscopy. These analyses are facilitated by the enlarged metal-support phase boundary due to the embedded nanoparticles, enhancing the reactivity between metal and support. ${ }^{33}$ Moreover, it is possible to prepare these as free-standing films (without a substrate) by employing sodium chloride $(\mathrm{NaCl})$ single crystal facets (mostly (001)) as substrates that can subsequently be removed by dissolution in water. More details of this process are given below.

Vacuum-based thin film deposition methods can be categorized into either physical vapor deposition (PVD) or chemical vapor deposition (CVD) techniques. In the former, energy is transferred to the material to be deposited so that it is evaporated or atomized. This can either occur thermally (thermal evaporation, electron beam (e-beam) evaporation or pulsed laser deposition (PLD)) or via sputtering using noble gas ions. In CVD, a chemical reaction of one or more precursor molecule(s) at the surface of the substrate is used to form the thin 
film.

Usually, these techniques are carried out in metal chambers featuring different flanges onto which the evaporators are mounted. However, using multiple deposition techniques in such chambers either requires a large number of ports or the use of tools to exchange the flanges because most vacuum connections rely on O-rings or gaskets being compressed by an externally applied force (usually via screws). This makes changes cumbersome and timeconsuming. Furthermore, the chamber volume usually is rather large, leading to long pumpdown times and a large surface area for adsorption of residual gases, which negatively affects the film purity for applications such as magnetron sputtering. As a way to overcome this lack of simplicity and flexibility, we present an ultra-flexible modular high vacuum chamber that is based on the Schott Duran flat flange system and glass recipients. In this concept, the O-rings are compressed and sealed by the external atmospheric pressure after pumpdown. Subsequently, this allows a completely tool-free assembly and modification process, enabling a high degree of flexibility by permitting rapid changes to the setup (for instance switching between different deposition sources), while at the same time maintaining a small volume and, thus, quick pump-downs. Additionally, the use of glass chambers guarantees clear/inert conditions and leads to an improved quality of the deposited films.

We demonstrate the exemplary deposition and characterization of a PVD-deposited $\mathrm{Pt} / \mathrm{ZrO}_{2}$ model catalyst system prepared in this chamber by means of transmission electron microscopy (TEM), X-ray photoelectron spectroscopy (XPS) and atomic force microscopy (AFM).

\section{THE MODULAR HIGH VACUUM CHAMBER}

Example configurations of the modular high vacuum chamber are given in Figure 1A and B. The photograph in A shows the setup for electron beam evaporation (the background was blurred to focus the attention on the chamber itself). The main components are the glass cross in the center, to which four different elements can be connected, and metal rings that can be used to feed media like gas or electrical power into the chamber. The glass and the other components are all based on flat flanges (more details are given below) that do not require any screws or similar hardware, but are rather sealed just by the force of the external pressure and gravity. This allows for rapid changes of the setup, as a benefit of 

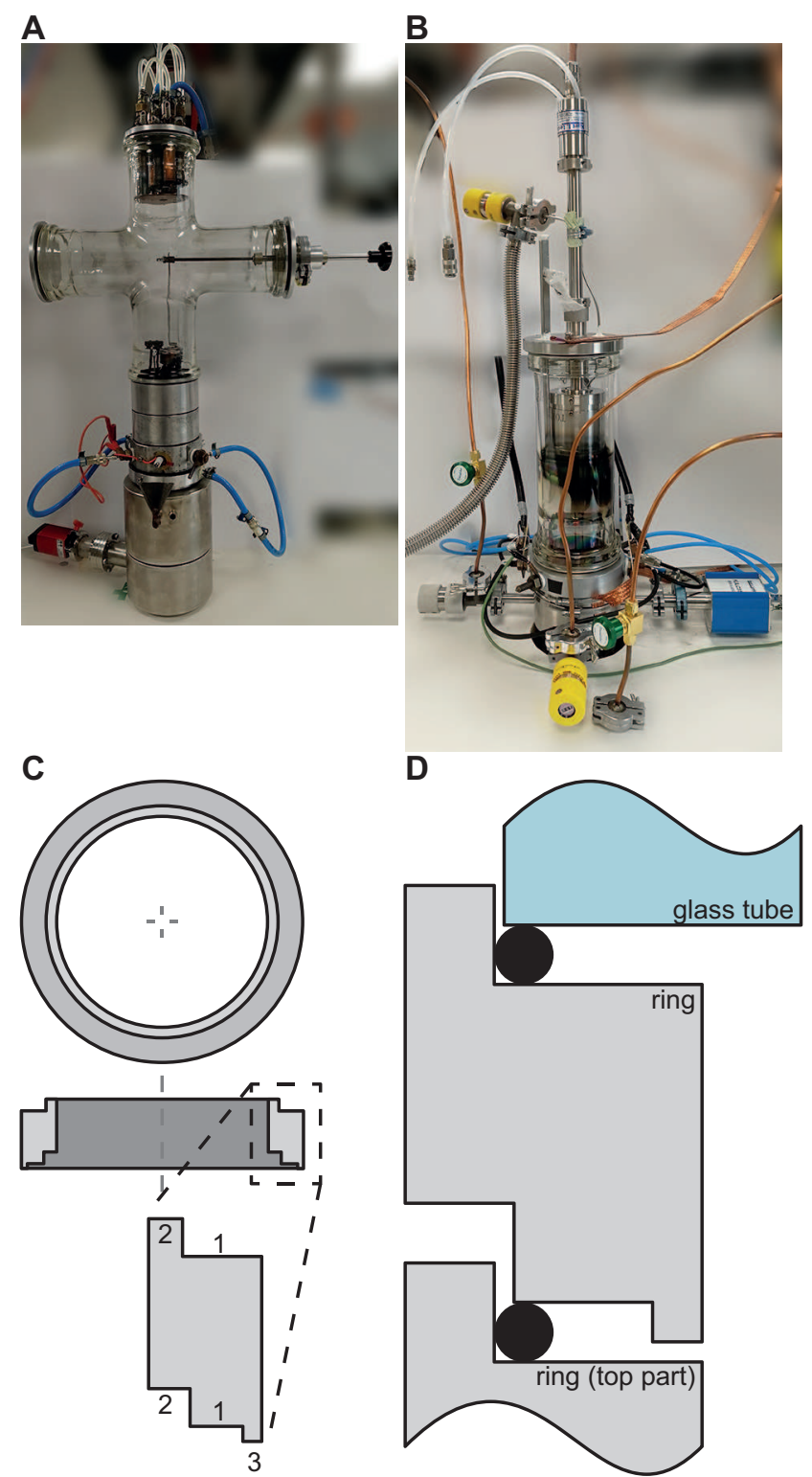

FIG. 1. A-B) Photographs of two modular high vacuum chamber configurations. C) Overview of the home-built stainless steel/aluminum ring modules that form the foundation of this chamber. Top: top view, bottom: cross section view of a ring (one side is enlarged below). 1 labels the sealing faces (see panel D), 2 the centering groove/ridge and 3 is a protective protrusion. D) Detailed view of a ring-ring-glass-tube assembly, including the O-rings (drawn as black circles). The wavy cut-offs denote that the respective elements are drawn only partially. The dimensions of these self-designed rings are given in Figure S1 in the supplementary material. 
the modular nature of this system. In this case, the left of the glass cross is blanked off by an aluminum lid. The right arm is fitted with a sodium chloride crystal cleaver (see Section III for more information regarding this). At the top, an electron beam evaporator is shown, which will also be explained later. Below the glass cross, there is a substrate holder with an integrated quartz crystal microbalance in order to monitor the deposition rates (and the film thickness). The larger ring with the integrated copper funnel is a liquid-nitrogenfilled cooling trap designed to improve the pressure while, at the same time, protecting the turbomolecular pump from deposition (i.e. it acts as a baffle) and the lowest ring in the picture contains a manometer. The turbomolecular pump and other fixed components are hidden below the table - all components shown in the photograph are simply attached by the suction of the vacuum and can be exchanged quickly and reproducibly.

The setup in Figure 1B is the one used for magnetron sputtering. It is based on exactly the same components and flanges as the other chamber. Here, a simple glass tube (showing a deposition layer on its inner surface due to the sputter process) is used instead of a cross, and it is located above a high temperature substrate holder, enabling temperatures of more than $1273 \mathrm{~K}$. This ring is subsequently placed on top of a ring containing two pressure gauges and two gas dosing valves to introduce additional gases into the chamber. The glass tube is covered by a flange housing the sputter source (a discussion of this flange is provided later) as well as the actuation mechanism of the shutter and an argon gas inlet (yellow valve on the top left).

The glass components are used for optical control of the processes in the chamber (with a better view than would be possible with a metal chamber containing multiple viewports). For instance, it provides an easy option to determine whether filaments are still intact. The glass recipients also allow for rapid prototyping of new equipment as potential collisions can be seen immediately when combining different elements. The metal rings (made of aluminum or stainless steel), on the other hand, provide a flexible choice regarding the elements attached to the chamber, such as gas inlets, electrical feedthroughs or evaporators.

In Figure 1C, one of these metal rings is shown in top view (upper part of the panel) and as a cross-section (bottom). These custom-made rings, which were designed to fit the standardized ground glass flat flanges from Schott (DN100), have an inner diameter of approximately $90 \mathrm{~mm}$ (see Figure S1 in the supplementary information for any details). Below, one side of the ring is drawn enlarged. The ring features three main elements, labeled 
1 through 3. The two surfaces denoted by "1" are the sealing faces of the ring, providing contact to the O-rings (refer to panel $\mathrm{C}$ and its discussion below). To aid the placement of the rings, the bottom part of the flange has a circular groove ("2") milled into it, while the top side has a corresponding ridge that fits into the groove of the next flange (or into the inner diameter of the glass tube). This automatically renders the ring/ring or ring/glass assembly centered and prevents the rings from accidentally sliding off each other. The small protrusion at the bottom that is labeled "3" serves as a protection for the lower sealing face for when the ring is placed on a surface, avoiding scratches that might compromise the ultimate pressure that can be reached.

This is displayed in more detail in Figure 1D, where a partial cross-section of a ring-ringglass assembly is drawn. In the lower region of this scheme, the arrangement of two metal rings placed upon each other is laid out. The function of the centering groove is apparent from this drawing, as well as the fact that the protective protrusion at the outer rim of the upper ring does not play any role when mounted to the chamber (i.e. this protrusion must be kept small enough so that it does not break the vacuum seal of the O-ring by touching the next ring below). The O-ring used here is a standard fluorocarbon ring and has an inner diameter of $99 \mathrm{~mm}$ so it can be placed around the outer side of the centering ridge. The next ring element then presses down upon this O-ring and the seal is formed by compression due to the force exerted by the vacuum itself. The influence of the latter is evident from the fact that crosses with horizontally oriented flanges can be used too (see Figure 1A). At the top of Figure 1D, the placement of the glass tube/cross is depicted. The glass tube, with an inner diameter of $100 \mathrm{~mm}$, fits perfectly around the centering ridge and consists of commercially available flat flanges (Duran Group, ground glass, DN100, without groove) that are attached to the ends of glass cylinders with wall thicknesses of $3 \mathrm{~mm}$. Hence, different source-to-substrate distances for the thin film deposition can be obtained by simply using a glass tube of another length. The easy exchange of these tubes thus makes it possible to use a clean recipient for every technique, avoiding cross-contaminations in the thin films in addition to having the optimized distance to the substrate for each deposition method.

The seal provided by the O-ring in these flanges is sufficient to easily reach pressures in the low $1 \times 10^{-7} \mathrm{hPa}$ regime, while still providing the big advantage of a tool-free assembly. Thus, these flanges cause no disadvantage in this regard when compared to other O-ringsealed flanges such as ISO-K or ISO-F ones. However, there is one drawback to these stacked 
flanges: since the different metal rings and glass components are not affixed to each other, there is the possibility of the whole construction tipping over if too many appendices are added to one side. However, this problem can be overcome by assembling the chamber in a way that the center of gravity remains as close as possible to the central axis of the rings/tubes (i.e. so that the whole assembly is roughly symmetrical). Moreover, the fact that glass crosses can be used (refer to Figure 1A) shows that more complex configurations are possible - once the system is evacuated, the external ambient pressure is sufficient to prevent it from falling apart. In fact, the only times care has to be taken to prevent the flanges from falling off is during the initial pump-down and when venting. While the usage of clamps would alleviate these problems to some extent, the time needed to (dis)assemble multiple clamps can become significant when exposure to air is to be minimized - even though the system is constantly flushed with argon, for air-sensitive samples, it is best to keep the chamber open for as short a time as possible. Also, in extreme cases, the whole chamber can be put inside a glovebag to maintain an inert environment. There, it becomes crucial to make the disassembly process easy enough to be handled with gloves (in addition to protecting the bag from potential ruptures due to additional metal objects).

The possible geometries of this chamber are not limited to simple glass tubes. As shown before (see Figure 1A), crosses can be used as well, allowing more complex setups. Furthermore, a Y-shaped glass tube can be used for co-deposition by employing, for instance, two electron beam evaporators to deposit two metals at the same time.

The vacuum and gas infrastructure surrounding the chambers is drawn in Figure 2. In our setup, there are two chambers (both based on the flanges detailed above and surrounded by dashed rectangles in the referenced scheme), where one is predominantly used for magnetron sputtering and the other one is used for other techniques, such as thermal evaporation or ion beam direct current sputtering. ${ }^{35,36}$ However, despite the first chamber being primarily dedicated to magnetron sputtering, it offers the same flexibility as the second one - the modular nature is a direct result of the flat flanges employed in the chamber.

As seen from Figure 2, both chambers are pumped by turbomolecular pumps (TMPs). In both cases, Leybold TURBOVAC 361 pumps with a nominal pumping speed of $350 \mathrm{~L} \mathrm{~s}^{-1}$ for Ar are employed. In our setup, both TMPs share a single roughing pump, a Leybold TRIVAC D16B rotary vane pump (RVP) with $16 \mathrm{~m}^{3} \mathrm{~h}^{-1}$. In both roughing lines, there are shut-off valves, so one chamber can be closed off while the other one is being evacuated 


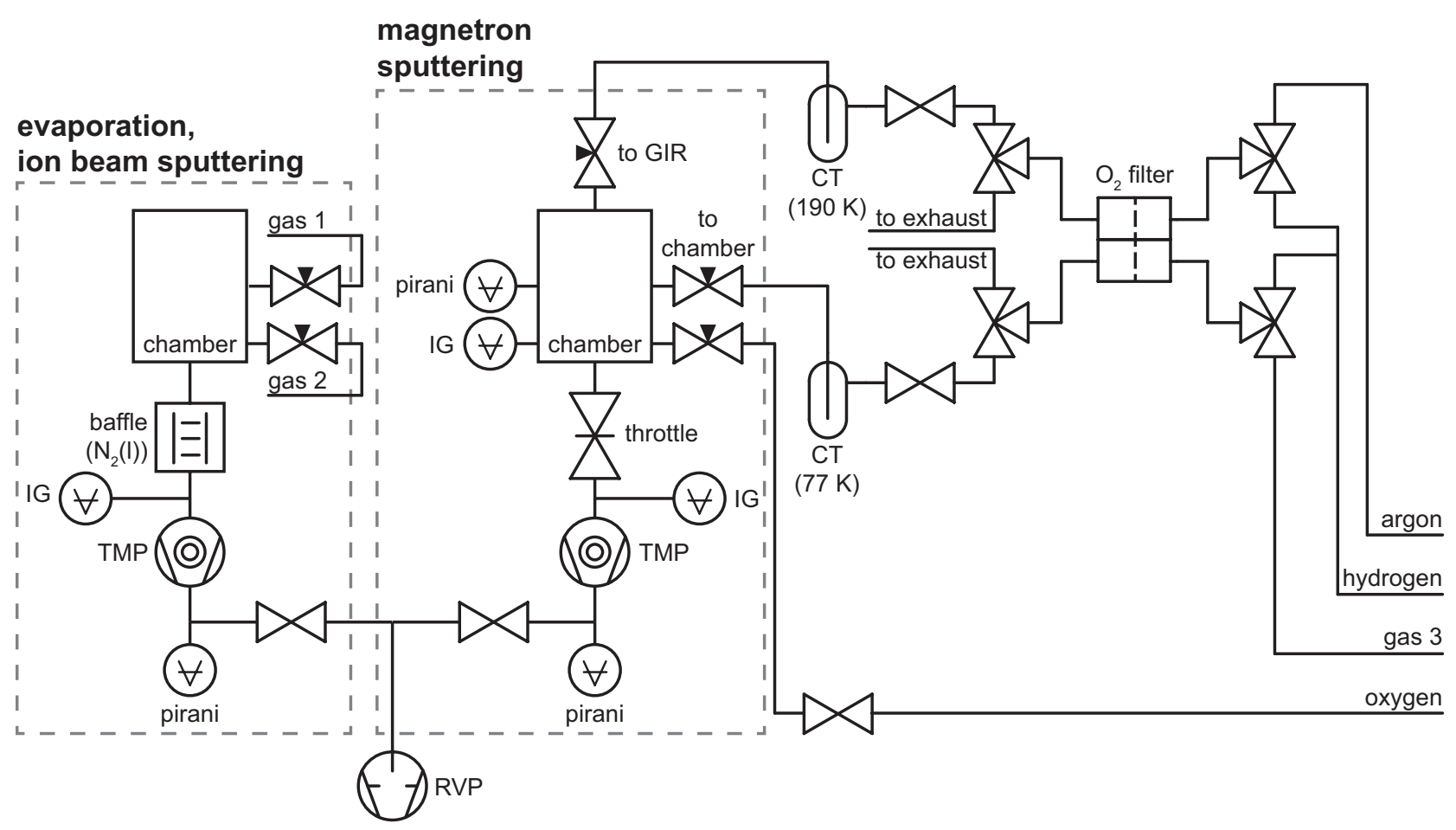

CT: cooling trap; GIR: gas injection ring; IG: ionization gauge; RVP: rotary vane pump; TMP: turbomolecular pump

FIG. 2. Gas and vacuum line layout of the modular HV system consisting of two chambers seen in Figure 1A and B that can be operated simultaneously, allowing for different techniques.

(to avoid venting the TMP due to the high load on the RVP). In case an oil-free system is desired, the roughing pump can easily be replaced by, for instance, a scroll pump. The roughing line also contains a pirani manometer to monitor the pre-vacuum. Above the TMP, both chambers feature a Bayard-Alpert-type ion gauge (IG).

The left chamber in Figure 2 contains a flat flange ring acting as a baffle to protect the TMP from deposited material. This baffle, containing a coiled copper tube, can also be cooled by liquid nitrogen. Thus, it can simultaneously act as a small cryo pump in order to freeze out water and other residual components and, as a consequence, improve the vacuum. Additionally, multiple gases can be introduced via gas dosing valves since lecture bottles (labeled "gas 1" and "gas 2") can be connected. This way, the Ar required by the ion beam sputter source, ${ }^{35}$ or oxygen that needs to be added for the deposition of certain reducible oxides such as ceria or tin oxide to maintain their fully oxidized state, ${ }^{37,38}$ can be added. The other components of the chamber, such as a ring that can be used as a thermal evaporator or a blank flange containing the e-beam evaporator, as well as the substrate holder, will be 
described later.

An example of the pressure evolution in this chamber during pump-down is given in Figure S2 in the supplementary materials. The time axis is measured relative to the time the TMP was turned on and the plot starts the moment the IG was turned on (just after the TMP reached its full speed), which was $120 \mathrm{~s}$ after the TMP was started. It can be seen that the pressure is already in the low $1 \times 10^{-5} \mathrm{hPa}$ range and is still decreasing. After less than two minutes, the baffle was filled with liquid nitrogen (see the arrow in the plot). This resulted in a radical pressure drop of about an order of magnitude due to the adsorption of gaseous species on its cooled walls. Less than 10 minutes after starting up the TMP, the pressure is already at approximately $5 \times 10^{-7} \mathrm{hPa}$.

The magnetron setup is more comprehensive regarding its components. First of all, the relatively high pressures (in the mid $1 \times 10^{-3} \mathrm{hPa}$ to the low $1 \times 10^{-2} \mathrm{hPa}$ range) required to ignite and maintain the Ar plasma would lead to too large gas loads for the TMP. Thus, a gate valve has to be used to throttle the pumping speed and, subsequently, the gas throughput. The chamber itself contains an IG and a pirani for pressure measurement, with the latter featuring a reasonably linear response in the desired pressure range to be of use for mixing multiple gases (for even more accurate results, a capacitance manometer can be employed). Additionally, for reactive sputtering, there are two gas dosing valves to introduce various gases into the chamber distant to the plasma (such as oxygen, see Section III). In contrast to feeding gases directly into the plasma, which could destroy/poison the target, here, the reaction is limited to the formed thin film. There is an additional gas port in the flange of the magnetron source (see its description below) that is connected to the gas injection ring (GIR) of the sputter source, which leads the gas (argon) directly to the sputter target. This facilitates the ignition and sustenance of the plasma at lower overall chamber pressures due to a locally higher concentration of Ar.

For the sputter-deposition of non-oxidic materials, it is desirable to remove any traces of oxygen from the argon. Thus, we installed an oxygen filter (Anoxy-cil, Cluzeau Info Labo) in the gas line, which contains copper powder that is heated to $623 \mathrm{~K}$ and that can be regenerated with hydrogen. As visible on the right-hand side of Figure 2, the filter has two separate inputs, i.e. two gases can be used simultaneously. Via three-way valves, the first input can be switched between argon and hydrogen (for the regeneration), and the second input between hydrogen (in order to remove the oxygen) and another gas for 
reactive sputtering (denoted by "gas 3", e.g. nitrogen or methane). For the third gas, the filter can also be bypassed completely and it can be connected to the chamber directly, like shown for oxygen. After the oxygen trap, another couple of three-way valves can be used to switch the output between the exhaust (needed to reach the high flow rates required for the regeneration and to purge the lines) and the chamber. Since water in the plasma can also have undesirable oxidation effects during sputtering of non-oxides, we also installed cooling traps (CTs) in front of the chamber. The CTs can be shut off to avoid contamination of the high-pressure side when they are warmed up. They are especially important when hydrogen is used because it constantly regenerates the oxygen filter, reducing the traces of copper oxide, thereby forming water. Due to the high boiling point of $\mathrm{Ar}(87.30 \mathrm{~K}),{ }^{39}$ its CT cannot be operated with pure liquid nitrogen (boiling point: $77.4 \mathrm{~K}$ ), ${ }^{39}$ as it would freeze out the argon; hence, it is cooled with an ethanol/nitrogen mixture at approximately $190 \mathrm{~K}$. For hydrogen, these problems do not occur and pure liquid nitrogen $(77 \mathrm{~K})$ can be used instead.

Not shown in Figure 2 are the baking possibilities that allow for further improvement of gas purity and vacuum. All gas pipings consist of copper tubes with an inner diameter of $4 \mathrm{~mm}$, apart from the copper cooling traps. These are also made out of coiled copper tubes but have been thermally insulated by short pieces of a stainless steel tube on either side. All gas lines can be baked with a heating tape to remove water residues. To facilitate this, multiple thermocouples (type K) have been installed along the lines in order to monitor (and regulate) the temperature at different locations during the bake-out process. Additionally, the magnetron chamber can also be baked out using an infrared (IR) lamp that is connected via an electrical feedthrough, which is mounted on a KF40 port below the throttling gate valve. Details on this component are given in Figure S3 in the supplementary material. As the pressure below the throttling valve remains low enough, the IR lamp can be replaced by a quadrupole mass spectrometer to monitor the gas composition during operation.

Since the adsorption of water on silica glass surfaces is very pronounced, ${ }^{40}$ it might be beneficial for different processes to get rid of these adsorbate layers prior to the deposition. Especially, as during magnetron sputtering, the presence of water in the plasma results in a strongly oxidative environment, ${ }^{41}$ potentially leading to oxide contaminations in the (nonoxidic) thin films. Since, in our case, the plasma is in close proximity to the inner walls of the glass tube (below, more details on how the sputter source is mounted will be given), we devised a portable and easy-to-mount self-made bakeout $u$ tensil for $g$ lass tubes (SMAUG). 

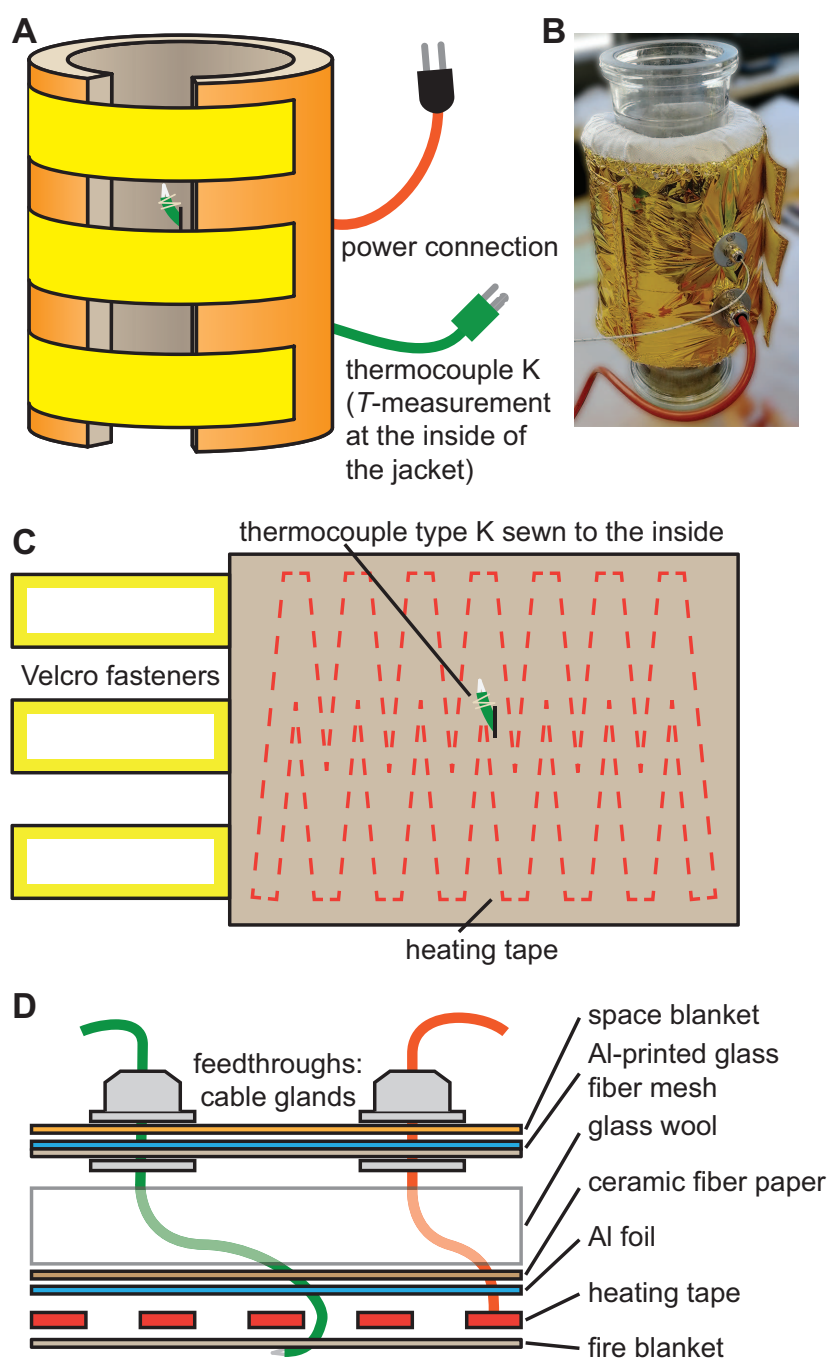

FIG. 3. The self-made bakeout $u$ tensil for $g$ lass tubes, SMAUG. A) 3D rendering, B) photograph of the backside, C) unwrapped view of the inner surface and D) cross section showcasing the different layers that make up the jacket.

Figure 3 depicts this heating jacket. In panel A, a 3D rendering is shown, where the Velcro fasteners that allow SMAUG to be tightened around the glass tube, preventing it from sliding down, can be seen (yellow stripes). At the backside, as visible in the photograph in panel B, there are two electrical feedthroughs (cable glands mounted to the outer layers, see below). One is powering a heating tape located inside the mantle and the second one connects the thermocouple that protrudes from the inner surface of the jacket. This thermocouple is used to measure and regulate the temperature of the glass surface during the baking process, which is essential in order not to destroy the magnets of the magnetron source. 
In the unfolded view of the inner surface of SMAUG in Figure 3C, the Velcro fasteners are drawn on the left-hand side. The layout of the heating tape, which forms the functional foundation of this baking equipment, is rendered schematically by the dashed outlines (red). It follows a zig-zag pattern in order to make the heat distribution to the glass surface as homogeneous as possible. This is further facilitated by the fact that the heating tape is sewn onto an $\mathrm{Al}$ sheet (with a thickness of $0.2 \mathrm{~mm}$ ) using heat-resistant glass fiber thread (from Vitcas). The $\mathrm{Al}$ foil acts as a heat reflector, directing the thermal radiation toward the chamber walls. Furthermore, its thermal conductivity also makes the temperature distribution more homogeneous along the circumference of the jacket. A schematic cross-section, listing the different materials that make up this mantle, is displayed in Figure 3D. The inner side is based on a fire blanket, which is made of a glass fiber mesh, yet still flexible enough to be suitable for this application. Onto the side facing the glass tube (bottom side in the graphic), the tip of the thermocouple is sewn to provide information about the current baking temperature (which is fed into a PID controller, keeping it constant). Next, there is the aforementioned heating tape that is affixed to the Al sheet. Behind this reflector shield, there is a ceramic fiber paper $(2 \mathrm{~mm})$ that forms the first stage of the thermal barrier. The main insulating material is composed of glass wool, which is surrounded by an Al-printed glass fiber mesh to further enhance the heat spread. In order to protect the brittle metal layer, we added an additional layer based on a space blanket (mounted with the reflective side facing the inside) to the outside to improve the handling. The cable glands for the power and thermocouple cables are fed through these outer two layers, with the respective wires and cables being led around the insulating material inside the jacket.

When SMAUG is heated to an interior temperature of $383 \mathrm{~K}$, the outside does not exceed $310 \mathrm{~K}$, rendering it safe to touch. Furthermore, a calibration of the temperature as measured using the thermocouple sewn to the inside of the baking jacket with respect to the temperature measured within the evacuated glass tube revealed there to be negligible differences $(<1 \mathrm{~K})$ after a very brief equilibration period in the order of one minute, despite the relatively high wall thickness of $3 \mathrm{~mm}$ and the low thermal conductivity of borosilicate glass ${ }^{42}$ underlining the effectiveness of the construction of SMAUG.

Since all three baking components (the bands for the gas lines, SMAUG and the IR lamp) are operated at a standard voltage of $230 \mathrm{~V}$ AC, we use a multi-channel dimmer pack (Botex DPX-620-3), as it is used for stage lighting, to power them. This provides 
an affordable solution to have a digitally controlled power supply, with each channel being regulated separately by temperature controllers.

The main component in the chamber devoted to magnetron sputtering (see Figure 2), is a Torus $2 \mathrm{HV}$ sputter source from Kurt J. Lesker Company that is designed to operate with targets of $50.8 \mathrm{~mm}$ in diameter. This source needs to be fitted with a shutter to enable pre-sputtering of the target, in order to get rid of contaminants on the sputtering target and, thus, improve the deposited thin film purity. However, since the head of the Torus is already more than $80 \mathrm{~mm}$ wide, the space inside the DN100 glass tube is very constricted, requiring a custom solution for the actuation mechanism. Our design is sketched in Figure 4. The stainless steel shaft of the source itself is mounted to a blank (flat) flange via a quick connect coupling in a slightly off-centered position (shifted by $5 \mathrm{~mm}$ in order to create more room for the shutter). On the right-hand side of the illustration, there is the Ar line feeding the gas injection ring (GIR), which provides a locally higher Ar pressure in the proximity of the target. On the left-hand side, the shutter mechanism is shown. The shutter (drawn in blue below the gun head) is a sheet of aluminum that was bent to a radius of $50 \mathrm{~mm}$ to fit to the glass wall in its opened state (rendered transparent with dashed outlines). This increases the area of deposition as it minimizes the shadowing effect during operation. The sheet is attached to a fork-like $\mathrm{Al}$ part that is mounted to the lower end of a round stainless steel tube (outer diameter $8 \mathrm{~mm}$ ). The tube has a slit cut into it from the bottom (on the left and right sides in the graphic) to allow for the rotary movement of the fork. The latter also contains a small protrusion with an eye at the top, through which a tantalum wire $(d=0.2 \mathrm{~mm})$ can be attached. This wire, colored in blue in Figure 4 , is responsible for the movement of the shutter as it is led through the round tube and the flange into a stainless steel tube with a square cross-section (and a width of $10 \mathrm{~mm}$ ), which is fixed to the air side of the flange. Inside this square tube (i.e. in vacuum), the other end of the Ta wire is mounted to a small sheet of stainless steel. An array of eight neodymium magnets $(5 \mathrm{~mm}$, four on each side) is glued onto this sheet with a vacuum-compatible epoxy resin, as can be seen at the top of the scheme. They are complemented by additional, larger magnets $(10 \mathrm{~mm})$ on the outside that can be moved in order to actuate the shutter mechanism (manually or by means of an electric motor), thus eliminating the need for a complex and expensive linear motion feedthrough. The magnets are laid out in two groups with differing polarity to reduce slipping of the moving magnets (see the color code in Figure 4). When the outer 


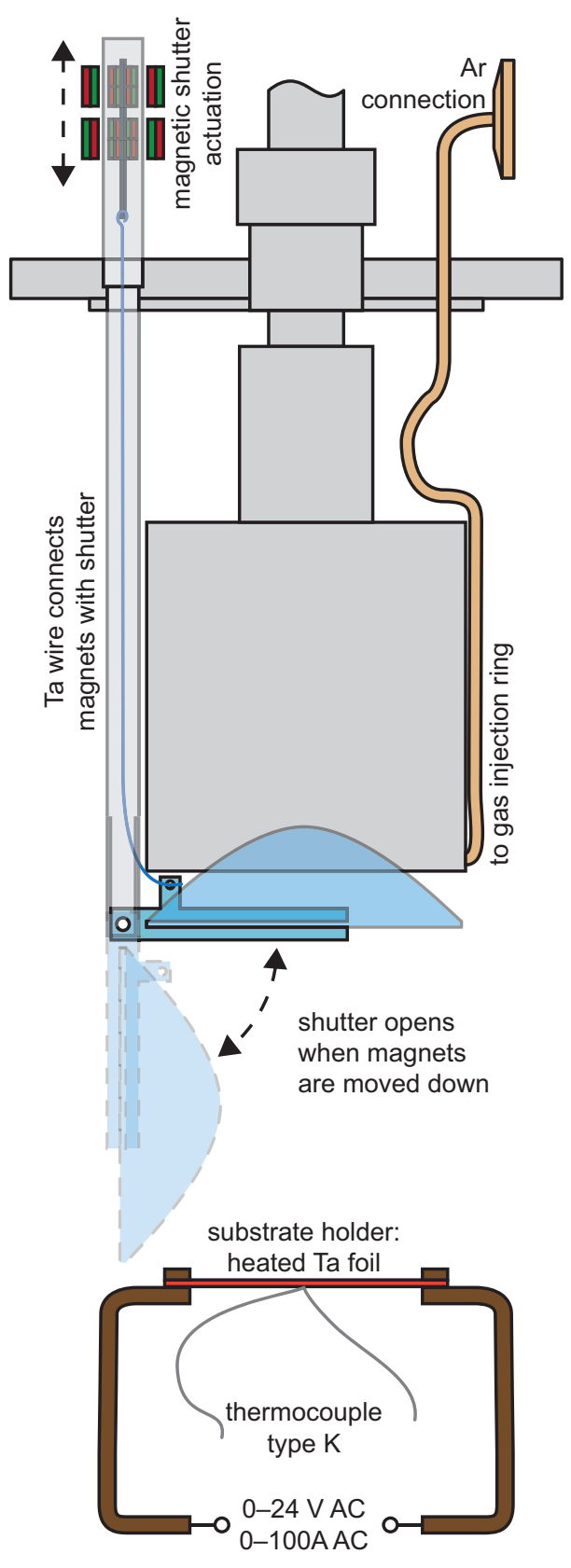

FIG. 4. The flange accommodating the magnetron sputter source (Torus $2 \mathrm{HV}$ by Kurt J. Lesker company), as well as the shutter mechanism, the argon supply and the high temperature substrate holder at the bottom. To make it more clear, the gas introduction is colored orange and the moving parts related to the shutter are rendered in blue. Some elements that would otherwise overlay other important parts were made transparent. 
magnets are moved down, the inner ones also move down and, by that, the Ta wire will push the shutter open (in fact, the shutter will stay in place in its closed state when the magnets are removed owing to the friction in the system). The reverse motion will close it again as the fork is hinged on the round tube by means of a copper screw.

The shutter is made of $\mathrm{Al}$ because of its low density. The reduced weight compared to a stainless steel plate enables the use of a thinner and more flexible Ta wire to connect the magnets with the shutter. Moreover, it has a lower sputter rate than similarly light materials, ${ }^{43}$ reducing contaminations on the target when pre-sputtering with a closed shutter.

In Figure 4, the high-temperature substrate holder is drawn below the sputter source. It is made out of a flat flange ring with electrical feedthroughs for power and a thermocouple, similar to the thermal evaporator detailed in Figure 5. It further consists of two copper brackets that are connected by a Ta foil (with a thickness of $0.1 \mathrm{~mm}$ ), which is heated resistively. By employing a power supply with an output of $100 \mathrm{~A}$ at voltages up to $24 \mathrm{~V}$, temperatures of more than $1273 \mathrm{~K}$ can be reached during the deposition. The copper brackets, which are water-cooled (not shown in the drawing), have the effect of keeping the Ta foil under tension when it is heated. Owing to the spring effect of this geometry, a sagging or buckling of the foil due to lowered tensile strength at high temperatures is prevented.

The radio frequency $(\mathrm{RF})$ power supply used for magnetron sputtering requires the chamber to be equipotential to its reference ground. To achieve this, braided copper cables $(15 \mathrm{~mm} \times 2 \mathrm{~mm})$ were connected to the magnetron flange via a tapped threaded hole, and to the other rings via hose clamps with a large diameter. These were set up in a way to press the copper ribbon onto the outside of the metal rings, forming an ohmic contact at each ring. This is necessary because, due to the tool-free assembly, the flat flange rings have no electric connection otherwise.

Finally, two alternative deposition sources will be described (see Figure 5), underlining the outstanding flexibility of the modular high vacuum chamber. Panel A shows the thermal evaporator, mounted to an $\mathrm{Al}$ ring, and in $\mathrm{B}$, the electron beam (e-beam) evaporator is drawn, which is fixed to a blank flange. In case of the thermal evaporator (Figure 5A), a boat made out of a highly refractory material, such as tungsten (W), tantalum (Ta) or molybdenum (Mo), is clamped to water-cooled copper blocks on both sides. These blocks, in turn, are connected to electrical feedthroughs. This element is then connected to a high-current transformer (NW, Schrack Wien) in order to heat up the evaporator boat 

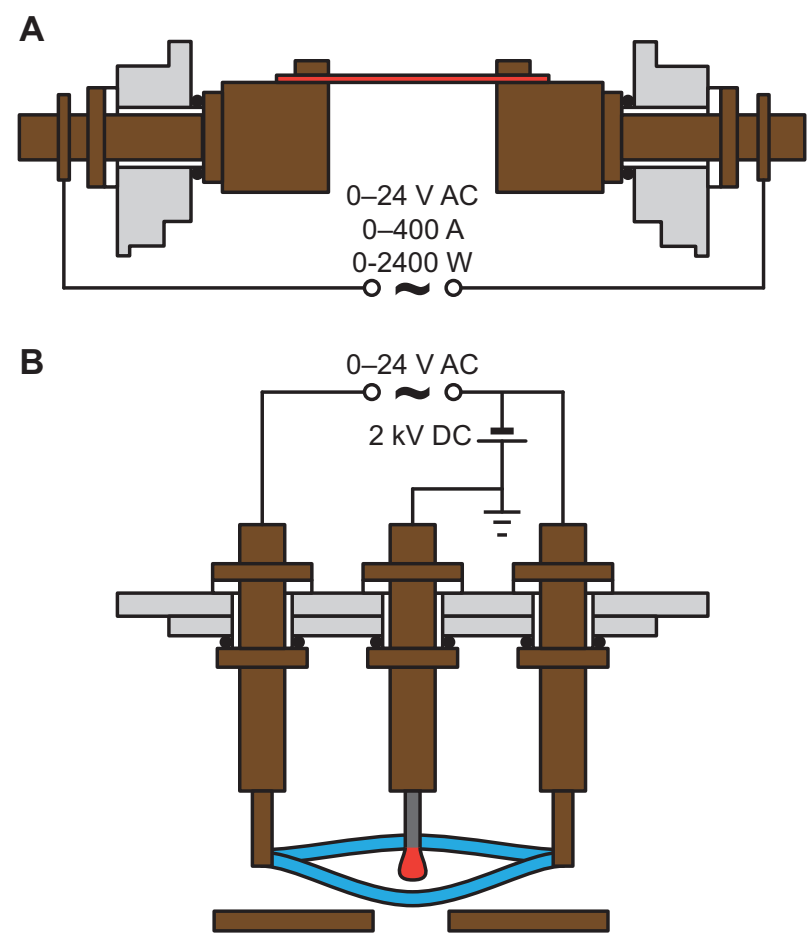

FIG. 5. Schematic depictions of the A) thermal and B) e-beam evaporators. In order to avoid cluttering of the graphic, the feedthroughs for the aperture of the e-beam evaporator are not drawn explicitly.

resistively. As rendered in the graphic, simple baseplate feedthroughs can be used, which are sealed by an O-ring and use PTFE as insulation materials. These only require a circular hole to be drilled into the $\mathrm{Al} /$ stainless steel ring and are rated to several hundred ampere and are, thus, ideal for this task. Alternatively, a $\mathrm{CF}$ half tube can be welded to a ring to allow the use of CF-based feedthroughs, which are able to handle higher temperatures as there are no O-ring sealings built in. This evaporator is suitable for primary oxides with no complex stoichiometry ${ }^{37}$ as, for instance, the composition of perovskites tends to change when evaporated and, hence, different methods are required. ${ }^{44}$ Moreover, materials with vapor pressures close to the boat materials are not suitable for this technique as the evaporation of those materials could not be prevented and would lead to contaminated specimens.

In the e-beam evaporator in Figure 5B, the material to be evaporated is a thin metal wire (about $0.125 \mathrm{~mm})$ that is wrapped around a $\mathrm{W} \operatorname{rod}(d=2 \mathrm{~mm})$. This rod is affixed to a baseplate feedthrough and is kept at ground level. Via two other feedthroughs, a filament 
made out of a $\mathrm{W}$ ribbon $(1.5 \mathrm{~mm} \times 0.05 \mathrm{~mm})$ is heated resistively. In addition, the whole filament assembly is raised to a potential of about $-1.5 \mathrm{kV}$ to $-2 \mathrm{kV}$ (with an $\mathrm{HV} 2.2$ power supply by Leybold AG). Thereby, the electrons are repelled from the filament and transfer their energy to the source material upon impact. Consequently, the wire melts and forms a drop at the end of the $\mathrm{W}$ rod, thus preventing the $\mathrm{W}$ itself from being evaporated. To prohibit contaminations from other elements of the evaporator, all feedthroughs are watercooled and an aperture is placed in front of the W rod (at the bottom of the illustration, its feedthrough is not drawn to avoid confusion). The aperture is placed in such a way that the $\mathrm{W}$ filament has no line of sight to the substrates, i.e. no evaporated (oxidic) W can contaminate the films.

\section{EXAMPLE DEPOSITION: PLATINUM/ZIRCONIA}

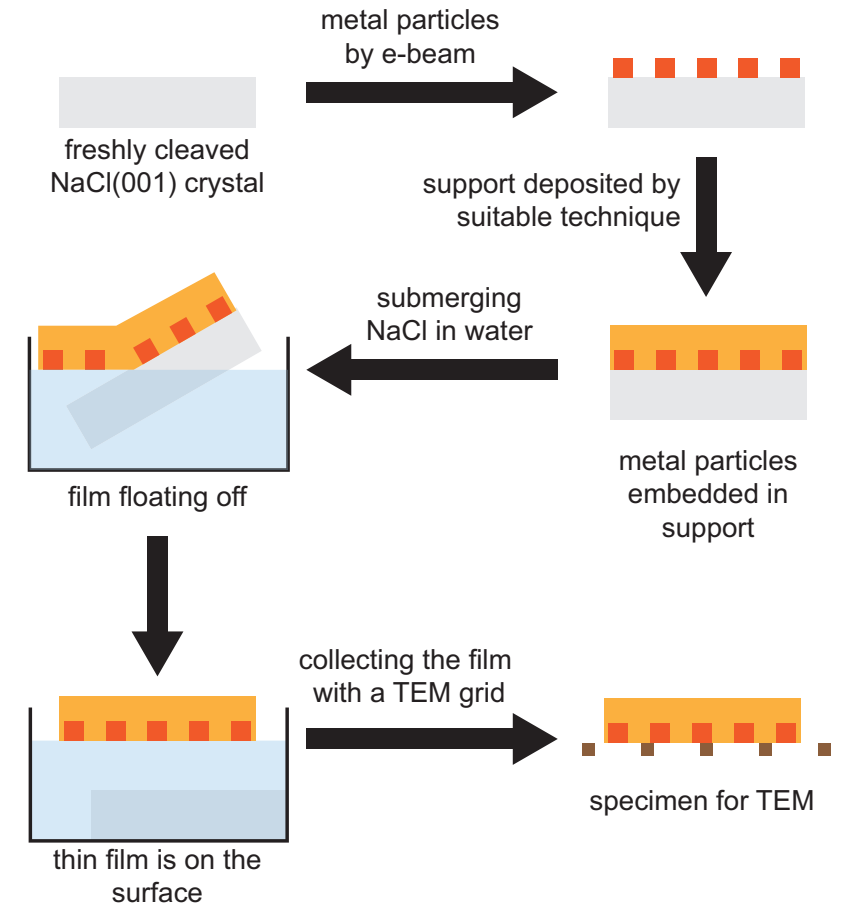

FIG. 6. The process of preparing free-standing thin films by floating them off the $\mathrm{NaCl}(001)$ substrates - shown for the example of metal particles being embedded in another material.

To showcase the capabilities of the modular high vacuum system presented in this work, we prepared an unsupported platinum/zirconia model catalyst thin film. This process is illustrated in Figure 6. As a substrate, a freshly cleaved sodium chloride $(\mathrm{NaCl})$ single 
crystal (Korth Kristalle $\mathrm{GmbH}$ ) is used, which can be removed afterwards by dissolution. For a metal/support system, metal particles are deposited first, usually by e-beam evaporation. During this initial step, the substrate is heated to at least $573 \mathrm{~K}$ to facilitate the ordered growth and crystallization of the particles. Next, these particles are covered by the support material. The latter can be deposited using different techniques, such as thermal evaporation, ${ }^{37,38}$ ion beam sputtering ${ }^{35,44,45}$ or magnetron sputtering. Here, the substrate temperature can control the degree of crystallinity of the support - for instance, at room temperature, the films usually become amorphous. If the substrate is then submerged in deionized water, the $\mathrm{NaCl}$ will start to dissolve and the thin film floats at the water/air interface. From there, it can be collected using a transmission electron microscopy (TEM) grid.

In the case of the $\mathrm{Pt} / \mathrm{ZrO}_{2}$ system, the $\mathrm{Pt}$ was first deposited onto pre-cleaved $\mathrm{NaCl}(001)$ substrates using electron beam evaporation at a substrate temperature of $573 \mathrm{~K}$. In this case, the $\mathrm{NaCl}$ single crystals were cleaved outside the vacuum, which leads to more irregular and disordered particles that, however, feature a significantly increased metal-support interface, which is useful, for example, to elucidate strong metal-support interaction (SMSI) effects. Alternatively, the crystals can be cleaved in vacuo using a cleaving device based on a stainless steel sheet mounted to a rotary feedthrough (which is seen on the right-hand side in Figure 1A and is used to hit the pre-scratched substrates, thereby cleaving them inside the chamber, as detailed in Figure S4 in the supplementary material), causing the formation of epitaxially grown, more regular particles due to a more pristine single crystal surface. The e-beam evaporation was carried out at a base pressure of around $1 \times 10^{-6} \mathrm{hPa}$ and the deposition rate was monitored using a quartz crystal microbalance. The deposited amount can be estimated to a layer thickness of approximately $1.6 \mathrm{~nm}$, which is expected to result in the formation of nanoparticles.

The $\mathrm{ZrO}_{2}$ was subsequently deposited by reactive $\mathrm{RF}$ magnetron sputtering of a metallic Zr target. The target was self-made from a Zr foil (Alfa Aesar, $99.99 \%$ purity, 0.125 mm in thickness) that was placed onto a $\mathrm{Cu}$ backing plate. To the gas injection ring, pure $\mathrm{Ar}$ was supplied, while the rest of the chamber was backfilled with oxygen to lead to a $1: 1$ composition of $\mathrm{Ar} / \mathrm{O}_{2}$ (amounting to a total pressure of $1.0 \times 10^{-2} \mathrm{hPa}$ ). The plasma was sustained at a power of $15 \mathrm{~W}$ to get a low deposition rate (the total deposition time was $17 \mathrm{~h}$ ), which is expected to produce crystalline and ordered thin films. Likewise, the substrate was 
kept at $573 \mathrm{~K}$. In addition to the $\mathrm{NaCl}$ single crystal, a silicon ( $\mathrm{Si}$ ) wafer was taken as a substrate for use in photoelectron spectroscopy measurements.

Both after the e-beam deposition and the sputtering, the chamber was vented with argon and constantly flushed with this inert gas during reassembly. Afterwards, the samples were briefly exposed to air during the transport to the spectrometer and the scooping of the $\mathrm{NaCl}$-grown films, which is not expected to have a big influence for this system. For more air-sensitive samples, the whole chamber can be placed inside an argon-filled glovebag to ensure inert conditions.

Figure 7 shows TEM results, which were obtained using a FEI Tecnai F20 S-TWIN microscope (operated at $200 \mathrm{kV}$ ). The bright field image in Figure 7A shows dark particles, which correspond to the Pt nanoparticles (see the high-angle annular dark field (HAADF) image below for confirmation, which features primarily mass contrast). There appear to be two main sizes of these particles: larger ones with approximately $3 \mathrm{~nm}$ in diameter that make up the majority of the image and smaller ones (in the range of $1 \mathrm{~nm}$ ) that are featured in the brighter areas that lack the larger ones. This is verified by panel $\mathrm{B}$, which contains the particle size histogram (obtained by measuring more than $100 \mathrm{Pt}$ particles). This histogram reveals a bimodal distribution with maxima at 3.5(1) nm and 1.4(3) nm, respectively, as obtained by fitting two Gaussian peaks. The smaller size could stem from Pt particles that just started nucleation. This could be a substrate effect as they appear to be confined to areas of several nanometer where no larger particles are found - i.e. they might have been unfavorable sites on the ex-situ-cleaved $\mathrm{NaCl}$ (a similar effect was observed for gold nanoparticles that preferentially nucleated at edge sites on graphite ${ }^{46}$ ). The bimodal size distribution additionally highlights the small and well defined particle sizes that are obtainable with this deposition method. Furthermore, it can lead to an extremely high platinum coverage of $92800 \mathrm{\mu m}^{-2}$ (measured by counting the Pt particles in a $50 \mathrm{~nm} \times 50 \mathrm{~nm}$ square), as is visible in the bright field image in Figure 7A.

The identity of the Pt particles could be affirmed using HAADF imaging, which primarily shows mass and thickness contrast. Thus, in the respective micrograph in Figure 7C, the bright regions correspond to $\mathrm{Pt}$ as its atomic mass $(195.078 \mathrm{u})$ is larger than the average molecular mass of $\mathrm{ZrO}_{2}(41.074 \mathrm{u}),{ }^{47}$ and thickness variations leading to such drastic contrast changes can be excluded for this type of specimen.

Crystallographic information about the specimen can be found in the right column of 
A

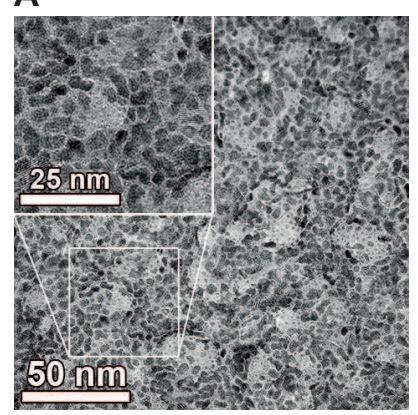

B

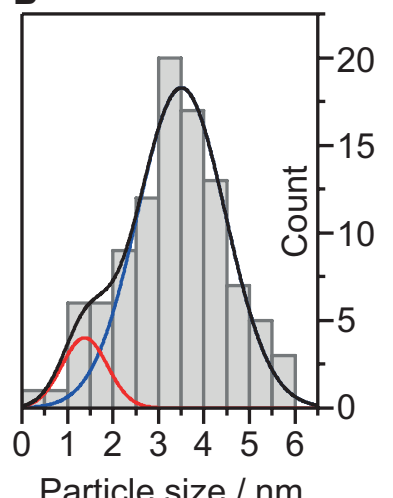

Particle size / nm

C

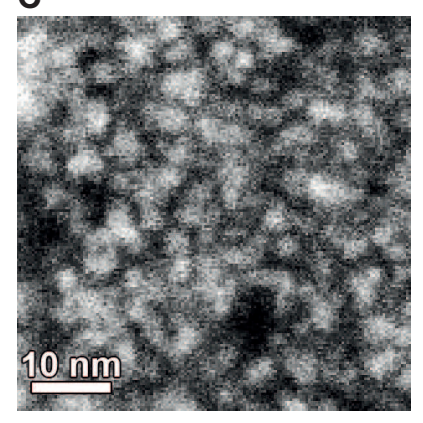

D
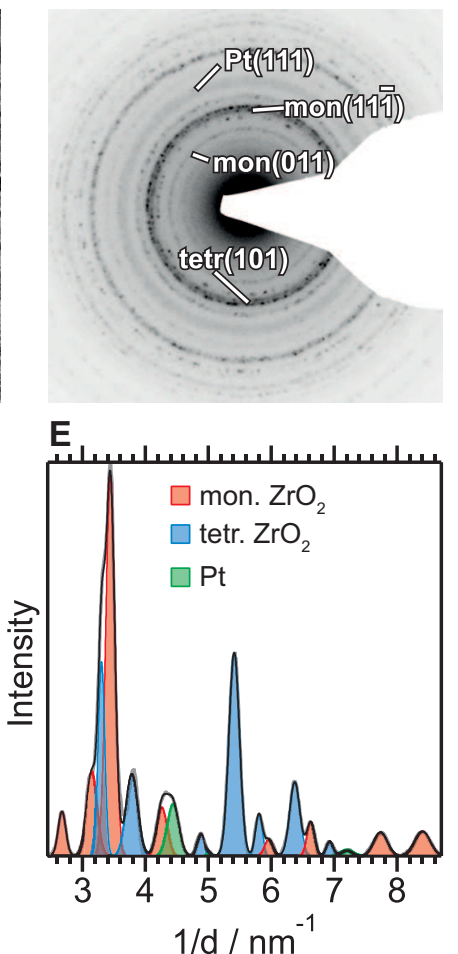

F

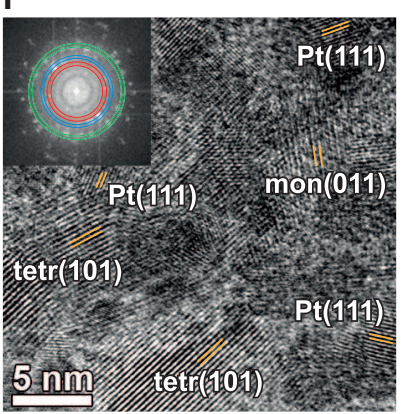

FIG. 7. Morphologic (A-C) and crystallographic (D-F) information on the $\mathrm{Pt} / \mathrm{ZrO}_{2}$ sample, acquired using TEM. A) Bright field image and magnified region (inset), B) histogram of the Pt particle sizes, C) high-angle annular dark field (HAADF) image. D) Diffraction pattern and its corresponding intensity profile in E), as well as a high-resolution TEM image in F). The inset shows the fast Fourier transform (FFT), where characteristic signals for each phase are marked by rings (with the same color coding as in E)).

Figure 7. A selected area electron diffraction (SAED) pattern is given in panel D. It exhibits diffraction rings typical for a disordered polycrystalline material. The assignments of some of the most characteristic rings are given in the image itself, while all other lattice spacings are listed in Table S1 in the supplementary material. The inner-most ring at $0.37 \mathrm{~nm}$ is due to 
the (001) planes of monoclinic $\mathrm{ZrO}_{2}$, which agrees well with the theoretical value calculated from the crystal structure - $0.369 \mathrm{~nm} .{ }^{48}$ Further signals from this phase are found at $0.32 \mathrm{~nm}$ (11), theoretical: $0.316 \mathrm{~nm}), 0.29 \mathrm{~nm}(111,0.284 \mathrm{~nm}$ according to the crystal structure) and $0.23 \mathrm{~nm}(021$, coinciding well with the predicted value of $0.233 \mathrm{~nm}) .{ }^{48}$ Another phase present is the tetragonal polymorph of $\mathrm{ZrO}_{2}$ : the rings at $0.30 \mathrm{~nm}$ (tetragonal (101) planes, with a theoretical spacing of $0.295 \mathrm{~nm}), 0.26 \mathrm{~nm}(002-0.259 \mathrm{~nm}), 0.20 \mathrm{~nm}$ (corresponding to the 102 reflex and predicted at $0.210 \mathrm{~nm})$ and $0.18 \mathrm{~nm}(112$, theoretically at $0.182 \mathrm{~nm})$ can be attributed to it. ${ }^{49} \mathrm{Pt}$ features lattice spacings very close to those of the two $\mathrm{ZrO}_{2}$ structures: its (111) planes are located in the widened ring at $0.23 \mathrm{~nm}$ (the peak position, as measured from an angular integration, is $0.225 \mathrm{~nm}$ ), which is in excellent agreement with the value obtained from the crystal structure $(0.226 \mathrm{~nm})$. Furthermore, the 200 reflection is found at $0.20 \mathrm{~nm}$ (compared to $0.196 \mathrm{~nm}$ theoretically). ${ }^{50}$

In contrast to previously deposited thin films of $\mathrm{ZrO}_{2}$ and yttria-stabilized zirconia (YSZ) that were prepared on the same substrates using thermal evaporation $\left(\mathrm{ZrO}_{2}\right)^{51}$ or a self-built direct current ion beam sputter source (YSZ), ${ }^{35,45}$ the thin film here is not purely tetragonal, but rather contains significant amounts of monoclinic $\mathrm{ZrO}_{2}$, despite the cubic substrate. This can most likely be attributed to the different deposition process and, consequently, the changed deposition mechanism during reactive sputtering. For instance, an oxidic target was used in the ion beam source (which was hit by a beam of argon ions), ${ }^{35,45}$ whereas a metallic one was employed in the present work (which was exposed to an RF argon plasma), where sputtered Zr species still need to react with oxygen from the chamber during deposition. Additionally, the target becomes much hotter in the ion beam sputter source than in the magnetron source.

The presence of the Pt signals is more obvious from the azimuthally integrated intensity profile depicted in Figure 7E. A series of Gaussian peaks, corresponding to the lattice planes of the three phases, was fitted to this profile. In the plot, these peaks are color-coded to show the structure. The broad peak with the plateau-like top at about $4.3 \mathrm{~nm}^{-1}$ can be deconvoluted into two peaks, the monoclinic 021 signal and the Pt 111 one. ${ }^{48,50}$

The area covered by the high-resolution TEM (HRTEM) image in Figure 7F contains all three phases: there are multiple Pt particles, as evident by the presence of Pt(111) lattice fringes, as well as patches of monoclinic (upper right) and tetragonal zirconia (bottom center). The inset in the upper-left corner shows the fast Fourier transform (FFT), where 
some of the characteristic lattice fringes are marked.

The $\mathrm{Pt} / \mathrm{ZrO}_{2}$ system was additionally deposited onto a $\mathrm{Si}(100)$ wafer to permit X-ray photoelectron spectroscopy (XPS) measurements. These were conducted in a Thermo Scientific MultiLab 2000 instrument, reaching base pressures in the low $1 \times 10^{-10} \mathrm{hPa}$ range. This spectrometer was fitted with an Alpha 110 hemispherical sector analyzer and a monochromated $\mathrm{Al} \mathrm{K}_{\alpha}$ X-ray source $(h \nu=1486.6 \mathrm{eV})$. Further equipment comprised a flood gun, providing electrons with kinetic energies of $6 \mathrm{eV}$ for charge compensation, and an ion source for sputter depth-profiling (emitting $\mathrm{Ar}^{+}$ions at $3 \mathrm{keV}$ ).

The survey spectrum (corresponding to the surface of the sample) in Figure 8 reveals the presence of signals from zirconium (shaded light blue), oxygen (orange), as well as carbon (grey) and hafnium (purple). Adventitious carbon is to be expected and it stems from the transfer to the spectrometer and, thus, its presence cannot be prevented, while hafnium is an inherent impurity in all zirconium products as the two elements cannot be separated very well due to similar properties because of the lanthanoid contraction (it is already present in the foil used as the target, the purity of which was labeled as "99.99\% excluding Hf"). However, no contaminations resulting from the deposition procedure are found, highlighting the suitability of this setup to prepare impurity-free specimens (within the detection limit of XPS). Figure 8B shows the compositional depth profile as obtained by quantifying the Zr 3d, O 1s, Pt 4f and Si 2p regions (while correcting for the different escape depths via the Gries formula ${ }^{52}$ ). This profile exhibits the expected behavior: at the surface, only $\mathrm{ZrO}_{2}$ is present, while the Pt signal appears close to the interface to the Si substrate. The film thickness can be estimated by fitting a sigmoid function to the silicon profile. Si yields the most reliable results in this case as the high $\mathrm{Pt}$ density displaces the $\mathrm{Zr}$ at the interface to the substrate and the wafer contains a native oxide layer, rendering the $\mathrm{O}$ signal unusable. Based on this procedure, the thickness is approximately $30 \mathrm{~nm}$.

Example spectra at the surface and at depths denoted by the vertical dashed lines in the depth profile are given in Figure 8C. At the surface (top-most spectrum), no Pt can be discerned in the $4 \mathrm{f}$ region, and the Zr 3d range can be described by only $\mathrm{Zr}(\mathrm{IV})$ species (the existence of a single state is further validated by the well-separated $3 \mathrm{~d}_{5 / 2}$ and $3 \mathrm{~d}_{3 / 2}$ peaks). ${ }^{53}$ Upon sputtering to a depth of $6 \mathrm{~nm}$, Pt signals are still not visible in the spectra, whereas the $\mathrm{Zr}$ region appears chemically in a slightly reduced state. In part, this is a measurement artifact as the Ar ion beam causes preferential sputtering of oxygen, thus reducing the 

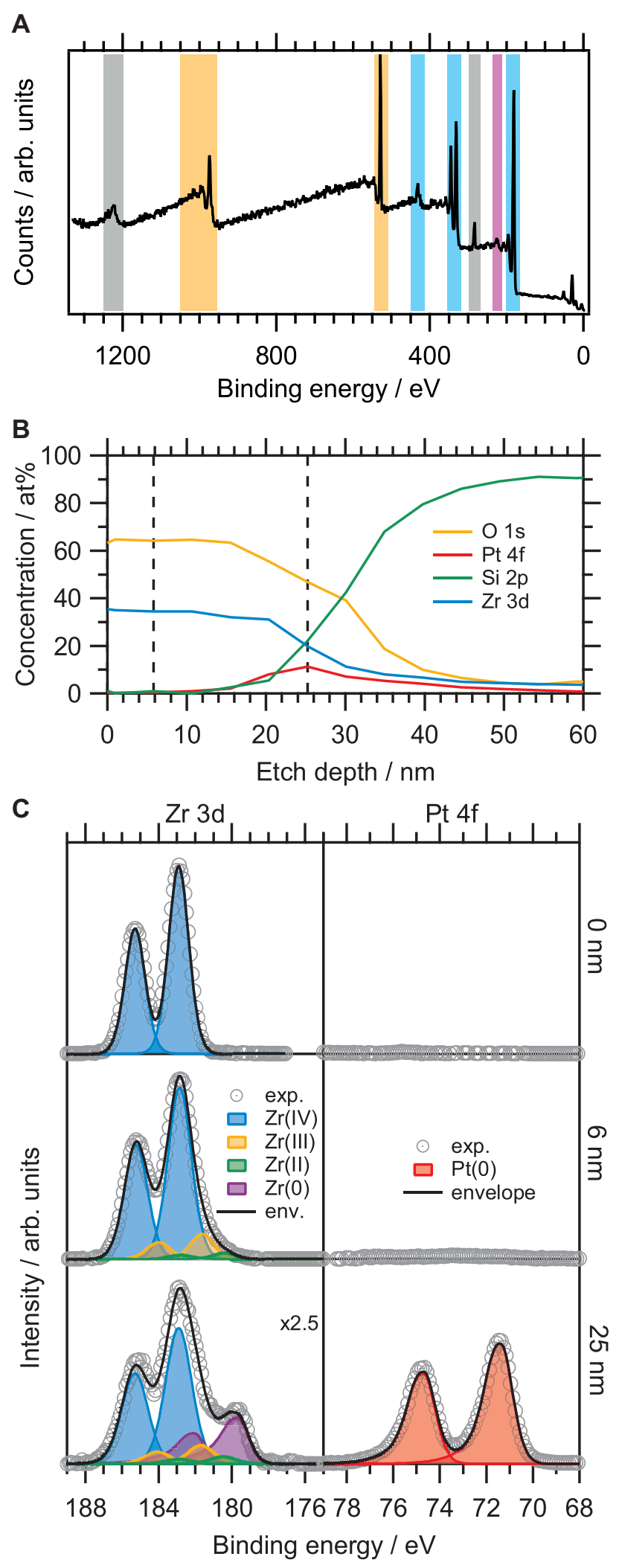

FIG. 8. A) XP survey spectrum of the thin film surface. B) Compositional depth profile obtained using sputter-depth profiling and XPS of a sample deposited onto a Si wafer. C) Selected Zr 3d and Pt $4 \mathrm{f}$ spectra at the depths indicated by the dashed lines in B). 
zirconia slightly. ${ }^{35}$ In addition to a low-binding-energy side shoulder, the separation of the main peaks has become less pronounced, owing to the $\mathrm{Zr}$ (III) state overlapping the minimum between the two $\mathrm{Zr}(\mathrm{IV})$ peaks. ${ }^{53}$ At a depth of $25 \mathrm{~nm}$, the $\mathrm{Pt}$ concentration peaks and the $\mathrm{Si}$ $2 p$ intensity starts to become more prominent. Pt is only present in its metallic state - the $4 \mathrm{f}_{7 / 2}$ component is located at a binding energy of $70.9 \mathrm{eV}$, which is in excellent agreement with literature data. ${ }^{5-56}$ Also, while the $\mathrm{Zr} 3 \mathrm{~d}$ region is more strongly reduced this close to the Si interface (there is a noticeable amount of metallic Zr, which could be a side-effect of the preferential sputtering of oxygen during depth-profiling), the majority of $\mathrm{Zr}$ is still in its fully oxidized state. This is in contrast to a previous study using an ion-beam-sputtered thin film on $\mathrm{Si}$, which was predominantly metallic in its bulk. ${ }^{36}$ However, less oxygen was present in the chamber and the mechanisms are different for these sources.

The surface topology of the unsupported film (on the TEM grid) was recorded by means of atomic force microscopy (AFM) using a Veeco Dimension 3100 microscope, equipped with NuNano Scout 350 probes featuring a nominal force constant of $42 \mathrm{~N} \mathrm{~m}^{-1}$ and a resonance frequency of $350 \mathrm{kHz}$. To avoid artifacts from the surface structure of the sample stage, the TEM grid was not placed onto the stage directly, but rather across a groove, so the film had no contact to the underlying metal at the investigated region. The height image of the $\mathrm{ZrO}_{2}$-terminated surface (i.e. the side without Pt particles) in Figure $9 \mathrm{~A}$ (with dimensions of $5 \mu \mathrm{m} \times 5 \mu \mathrm{m})$ reveals that the surface, spanning a total $z$ range of $0.47 \mu \mathrm{m}$, consists of spherical grains. The visibility of these grains is improved in the phase image in panel B, which consequently is more suited to measure their sizes. ${ }^{57}$ In order to make the granular structure more clear, the marked areas $(1.5 \mu \mathrm{m} \times 1.5 \mu \mathrm{m})$ were enlarged in Figure 9C and D. By measuring the diameter of more than 100 of such grains, the histogram in panel $\mathrm{E}$ could be obtained. Similarly to the Pt particles, this shows a bimodal distribution, with maxima at $109(5) \mathrm{nm}$ and $55(8) \mathrm{nm}$. Because the zirconia-terminated surface of the thin film was analyzed using AFM, these sizes correspond to the $\mathrm{ZrO}_{2}$ grains, which are significantly larger than those of the Pt particles (measured using TEM data). The round surface morphology of these grains is typical for films obtained by reactive magnetron sputtering. ${ }^{58-61}$ Using Gwyddion, ${ }^{62}$ the RMS surface roughness, $S_{q}$, of the full-sized image could be calculated to be $56.7 \mathrm{~nm}$. At $19.7 \mathrm{~nm}$, it is significantly lower in the cropped region, owing to the lack of the large-scaled features in this flat area of the specimen. 

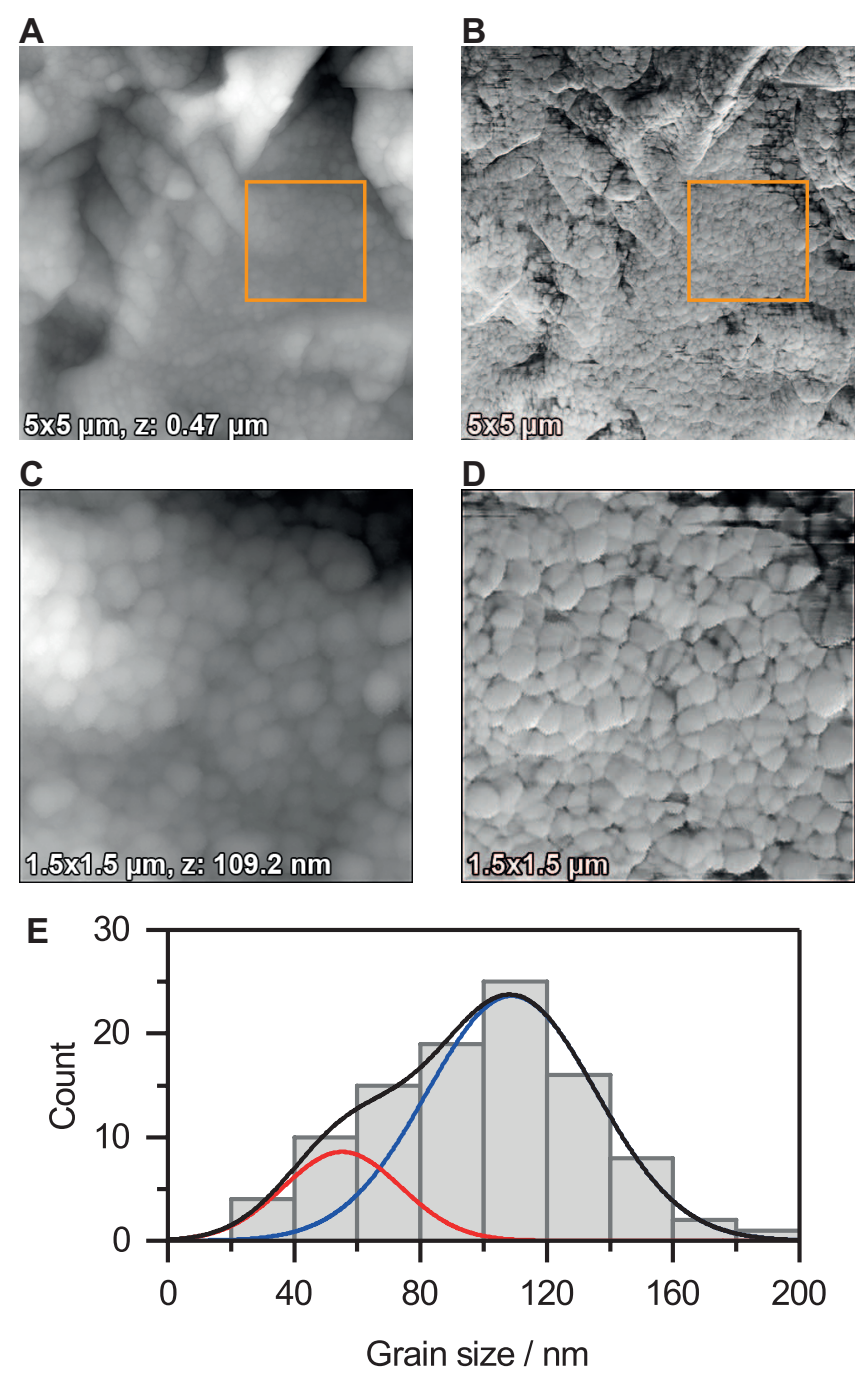

FIG. 9. A-D) AFM topology (A,C) and phase (B,D) images (corresponding to the original vacuum side during the deposition) of the free-standing sample on the TEM grid, acquired in tapping mode. Panels C) and D) show an enlarged version of the marked region in these micrographs. Note that the contrast in the phase images was modified to improve the clarity of the grains. E) The histogram of the grain size distribution, as measured from the AFM data, shows a bimodal distribution.

\section{CONCLUSION}

We have described a modular high vacuum chamber where the different elements are simply stacked onto each other. The sealing is based on flat flanges, which permit the complete restructuring of the system without the need for a single tool. This way, a high degree of flexibility is obtained: the compact chamber, providing quick pump-downs and small volumes, can be adapted to different thin film deposition techniques within minutes. 
As a consequence, multi-layer systems or nanoparticles embedded in a support can be easily prepared by simply switching between different deposition sources. This was demonstrated by the preparation and characterization of a $\mathrm{Pt} / \mathrm{ZrO}_{2}$ system, where Pt nanoparticles were synthesized by e-beam deposition and $\mathrm{ZrO}_{2}$ was deposited by reactive magnetron sputtering in $\mathrm{Ar} / \mathrm{O}_{2}$ (using an RF source).

The setup enables to introduce oxygen- and water-free gases into the chamber as the gas lines for the magnetron system are equipped with an oxygen filter and cooling traps. Furthermore, the lines, as well as the chamber itself, can be baked. For the chamber, we devised a self-made bakeout untensil for glass tubes ("SMAUG"), which is a heating jacket that can be wrapped around the glass tube.

While this manuscript focuses on the PVD-preparation of thin films, the flexibility of this chamber enables a multitude of other usages: gaseous precursors can be admitted into the chamber via gas dosing valves to perform CVD, and the thermal evaporator can be utilized to co-melt metals to form alloys and intermetallic compounds under the exclusion of oxygen..$^{63,64}$

\section{SUPPLEMENTARY MATERIAL}

See the supplementary material for the dimensions of the custom-made, non-standard flat flanges that make up the home-built and individually designed modular components of this chamber, a drawing of the IR baking lamp as well as details on the phase assignment to the SAED pattern.

\section{ACKNOWLEDGMENTS}

This work was financially supported by the Austrian Science Fund (FWF) via grant F4503-N16 and has been performed within the framework of the Forschungsplattform Ma-

terials and Nanoscience at the University of Innsbruck. T.G. and E.-M.W. acknowledge additional funding via FWF project I2877-N34. 


\section{REFERENCES}

${ }^{1}$ C.-C. Lee, in The Current Trends of Optics and Photonics, Topics in Applied Physics, Vol. 129, edited by C.-C. Lee (Springer Netherlands, Dordrecht, 2015) pp. 3-33.

${ }^{2}$ H. Bach and D. Krause, Thin Films on Glass (Springer Berlin Heidelberg, Berlin, Heidelberg, 2003).

${ }^{3}$ A. Piegari and F. Flory, Optical Thin Films And Coatings: From Materials To Applications, Woodhead Publishing Series in Electronic and Optical Materials (Elsevier Science, Burlington, 2013).

${ }^{4}$ M. Kamalisarvestani, R. Saidur, S. Mekhilef, and F. S. Javadi, Renewable Sustainable Energy Rev. 26, 353 (2013).

${ }^{5}$ N. Selvakumar and H. C. Barshilia, Sol. Energy Mater. Sol. Cells 98, 1 (2012).

${ }^{6}$ W. D. Sproul, Surf. Coat. Technol. 81, 1 (1996).

${ }^{7}$ J. M. Lackner and W. Waldhauser, J. Adhes. Sci. Technol. 24, 925 (2010).

${ }^{8}$ J. Laconte, D. Flandre, and J.-P. Raskin, Micromachined Thin-Film Sensors for SOICMOS Co-Integration (Springer US, 2006).

${ }^{9}$ K. Sahner and H. L. Tuller, J. Electroceram. 24, 177 (2010).

${ }^{10}$ T. Kamiya, B. Monemar, H. Venghaus, Y. Yamamoto, and Y. Hamakawa, Thin-Film Solar Cells, Vol. 13 (Springer Berlin Heidelberg, Berlin, Heidelberg, 2004).

${ }^{11}$ K. L. Chopra, P. D. Paulson, and V. Dutta, Prog. Photovolt: Res. Appl. 12, 69 (2004).

${ }^{12}$ R. B. Bergmann, Appl. Phys. A: Mater. Sci. Process. 69, 187 (1999).

${ }^{13}$ M. Kemell, M. Ritala, and M. Leskelä, Crit. Rev. Solid State Mater. Sci. 30, 1 (2005).

${ }^{14}$ D. Mount (Society of Vacuum Coaters, 2016) pp. 33-34.

${ }^{15}$ C. K. Witham, Electrochem. Solid-State Lett. 3, 497 (1999).

${ }^{16}$ M. S. Wilson and S. Gottesfeld, J Appl Electrochem 22, 1 (1992).

${ }^{17}$ A. Atkinson, S. Barnett, R. J. Gorte, J. T. S. Irvine, A. J. McEvoy, M. Mogensen, S. C. Singhal, and J. Vohs, Nat. Mater. 3, 17 (2004).

${ }^{18}$ A. Evans, M. Prestat, R. Tölke, Schlupp, M. V. F., L. J. Gauckler, Y. Safa, T. Hocker, J. Courbat, D. Briand, de Rooij, N. F., and D. Courty, Fuel Cells 12, 614 (2012).

${ }^{19}$ T. Götsch, T. Schachinger, M. Stöger-Pollach, R. Kaindl, and S. Penner, Appl. Surf. Sci. 402, 1 (2017).

${ }^{20}$ Y. Chen and M. W. Kanan, J. Am. Chem. Soc. 134, 1986 (2012). 
${ }^{21}$ T. Reier, I. Weidinger, P. Hildebrandt, R. Kraehnert, and P. Strasser, ECS Trans. 58, 39 (2013).

${ }^{22}$ H. Chang, Water Res. 34, 407 (2000).

${ }^{23}$ M. E. Zorn, D. T. Tompkins, W. A. Zeltner, and M. A. Anderson, Environ. Sci. Technol. 34, $5206(2000)$.

${ }^{24}$ P. Jain and E. J. Rymaszewski, Thin-Film Capacitors for Packaged Electronics (Springer US, Boston, MA, 2004).

${ }^{25}$ R. Waser, Integr. Ferroelectr. 15, 39 (2006).

${ }^{26}$ S. D. Brotherton, Introduction to Thin Film Transistors (Springer International Publishing, Heidelberg, 2013).

${ }^{27}$ Y. K. Hong, N. Liu, D. Yin, S. Hong, D. H. Kim, S. Kim, W. Choi, and Y. Yoon, J. Phys. D: Appl. Phys. 50, 164001 (2017).

${ }^{28}$ E. Fortunato, P. Barquinha, and R. Martins, Adv. Mater. 24, 2945 (2012).

${ }^{29}$ J. S. Park, W.-J. Maeng, H.-S. Kim, and J.-S. Park, Thin Solid Films 520, 1679 (2012).

${ }^{30}$ J. M. Daughton, Thin Solid Films 216, 162 (1992).

${ }^{31}$ J. Robertson, Thin Solid Films 383, 81 (2001).

${ }^{32}$ J. F. Scott and M. Dawber, Ferroelectrics 265, 119 (2002).

${ }^{33}$ S. Penner, Thin Solid Films 562, 1 (2014).

${ }^{34}$ M. Y. Smirnov, A. V. Kalinkin, A. V. Pashis, I. P. Prosvirin, and V. I. Bukhtiyarov, J. Phys. Chem. C 118, 22120 (2014).

${ }^{35}$ T. Götsch, W. Wallisch, M. Stöger-Pollach, B. Klötzer, and S. Penner, AIP Adv. 6, 25119 (2016).

${ }^{36}$ T. Götsch, B. Neumann, B. Klötzer, and S. Penner, Surf. Sci. accepted (2018).

${ }^{37}$ T. Götsch, M. Stöger-Pollach, R. Thalinger, and S. Penner, J. Phys. Chem. C 118, 17810 (2014).

${ }^{38}$ T. Götsch, K. Ploner, J. Bernardi, L. Schlicker, A. Gili, A. Doran, A. Gurlo, and S. Penner, J. Solid State Chem. 265, 176 (2018).

${ }^{39} \mathrm{C}$. R. Hammond, in CRC Handbook of Chemistry and Physics, edited by D. R. Lide (CRC Press/Taylor \& Francis, Boca Raton, FL, 2008).

${ }^{40}$ R. I. Razouk and A. S. Salem, J. Phys. Chem. 52, 1208 (1948).

${ }^{41}$ L. Pranevicius, S. Tuckute, L. L. Pranevicius, and K. Gedvilas, Acta Phys. Pol. A 123, 907 (2013). 
${ }^{42}$ H. P. R. Frederikse, in CRC Handbook of Chemistry and Physics, edited by D. R. Lide (CRC Press/Taylor \& Francis, Boca Raton, FL, 2008).

${ }^{43}$ Y. Yamamura and H. Tawara, At. Data Nucl. Data Tables 62, 149 (1996).

${ }^{44}$ T. Götsch, D. Hauser, N. Köpfle, J. Bernardi, B. Klötzer, and S. Penner, Appl. Surf. Sci. 452, 190 (2018).

${ }^{45}$ T. Götsch, L. Mayr, M. Stöger-Pollach, B. Klötzer, and S. Penner, Appl. Surf. Sci. 331, $427(2015)$.

${ }^{46}$ C. E. Cross, J. C. Hemminger, and R. M. Penner, 23, 10372 (2007), journal Article, 17715955 .

${ }^{47}$ R. D. Loss, Pure Appl. Chem. 75, 1107 (2003).

${ }^{48}$ D. K. Smith and W. Newkirk, Acta. Cryst. 18, 983 (1965).

${ }^{49}$ B. Bondars, G. Heidemane, J. Grabis, K. Laschke, H. Boysen, J. Schneider, and F. Frey, J. Mater. Sci. 30, 1621 (1995).

${ }^{50}$ K. H. Hong, G. M. McNally, M. Coduri, and J. P. Attfield, Z. Anorg. Allg. Chem. 642, 1355 (2016).

${ }^{51}$ R. Thalinger, M.-S. Pollach, B. Klötzer, and S. Penner, Mater. Chem. Phys. 138, 384 (2013).

${ }^{52}$ W. H. Gries, Surf. Interface Anal. 24, 38 (1996).

${ }^{53}$ Y. Nishino, A. R. Krauss, Yuping Lin, and D. M. Gruen, J. Nucl. Mater. 228, 346 (1996).

${ }^{54}$ D. Shuzhong, X. Fanhua, and D. Jingfa, J. Catal. 109, 170 (1988).

${ }^{55}$ Y. Baer, P. F. Hedén, J. Hedman, M. Klasson, C. Nordling, and K. Siegbahn, Phys. Scr. 1, 55 (1970).

${ }^{56}$ J. E. Drawdy, G. B. Hoflund, S. D. Gardner, E. Yngvadottir, and D. R. Schryer, Surf. Interface Anal. 16, 369 (1990).

${ }^{57}$ E.-M. Köck, M. Kogler, T. Götsch, L. Schlicker, M. F. Bekheet, A. Gurlo, A. Doran, B. Kloetzer, B. Petermüller, D. Schildhammer, N. Yigit, and S. Penner, Dalton Trans. (2017), 10.1039/c6dt04847a.

${ }^{58}$ B. Coskun, T. Asar, U. Akgul, K. Yildiz, and Y. Atici, Ferroelectrics 502, 147 (2016).

${ }^{59}$ P. Gao, L. Meng, M. dos Santos, V. Teixeira, and M. Andritschky, Vacuum 56, 143 (2000).

${ }^{60}$ R. Nédélec, S. Uhlenbruck, D. Sebold, V. Haanappel, H.-P. Buchkremer, and D. Stöver, J. Power Sources 205, 157 (2012). 
${ }^{61}$ S. Sønderby, A. J. Nielsen, B. H. Christensen, K. P. Almtoft, J. Lu, J. Jensen, L. P. Nielsen, and P. Eklund, Surf. Coat. Technol. 206, 4126 (2012).

${ }^{62}$ D. Nečas and P. Klapetek, Open Phys. 10, 99 (2012).

${ }^{63}$ N. Köpfle, L. Mayr, D. Schmidmair, J. Bernardi, A. Knop-Gericke, M. Hävecker, B. Klötzer, and S. Penner, Catalysts 7, 53 (2017).

${ }^{64}$ N. Köpfle, T. Götsch, M. Grünbacher, E. A. Carbonio, M. Hävecker, A. Knop-Gericke, L. Schlicker, A. Doran, D. Kober, A. Gurlo, S. Penner, and B. Klötzer, Angew. Chem. Int. Ed. Engl. 57, 1 (2018). 